\title{
NOTES
}

\section{RIFAMYCIN G, A FURTHER PRODUCT OF NOCARDIA MEDITERRANEI METABOLISM}

\section{Giancarlo Lancini and Giuseppe Sartori}

Gruppo Lepetit S.p.A., Research Laboratories, 20158 Milano, Italy

(Received for publication January 16, 1976)

Several natural rifamycins have been isolated in addition to the products of Nocardia mediterranei fermentation originally described by SENSI and coworkers. ${ }^{1)}$ These include rifamycin $\mathrm{SV}^{2)}$ and rifamycin $\mathrm{W}^{3)}$ obtained from mutant strains of $N$. mediterranei, rifamycin $\mathrm{L}^{4)}$ a microbial transformation product of rifamycin $\mathrm{S}$ and rifamycin $\mathrm{O}^{5)}$ produced by a Streptomyces isolated from a soil sample; the production of rifamycin SV from a Nocardia isolated from soil was also reported. ${ }^{\text {) }}$

We wish to report the isolation of rifamycin $\mathrm{G}$, a further product of $N$. mediterranei metabolism. Evidences for its origin from rifamycin $\mathrm{S}$ are also given and a structure based on physico-chemical determinations is proposed (Fig. 1). b) Separation from rifamycin complex by reextraction in buffer $\mathrm{pH} 7.38$.

c) Acidification and extraction with ethyl acetate.

d) Evaporation of the solvent and precipitation in hexane.

Purification was accomplished by counter current distribution (solvent system: $0.07 \mathrm{M}$ phosphate buffer-ethyl acetate) and crystallization from $95 \%$ ethanol.

Rifamycin $\mathrm{G}$ is a white crystalline powder m.p. $250 \sim 252^{\circ} \mathrm{C}$; u.v. absorption (in phosphate buffer $\mathrm{pH} 7.38): \lambda_{\max } 268 \mathrm{~nm}\left(\mathrm{E}_{1 \mathrm{~cm}}^{1 \%} 338\right), \lambda_{\max }$ $365 \mathrm{~nm}$ (shoulder), $\lambda_{\max } 383 \mathrm{~nm}\left(\mathrm{E}_{1 \mathrm{~cm}}^{1 \%} 234\right)$; two acidic functions with $\mathrm{pKa}$ values of 3.1 and 10.3 were calculated from variation of u.v. absorption with $\mathrm{pH}$. It analyses best for $\mathrm{C}_{36} \mathrm{H}_{47} \mathrm{NO}_{12} \cdot \mathrm{H}_{2} \mathrm{O}$ (M.W. 703.741, in agreement with the molecular ion in mass spectrum at $m / e=685)$.

\section{Origin from Rifamycin $\mathbf{S}$}

Rifamycin $G$ appears to be a metabolic derivative of rifamycin $\mathrm{S}$. The latter is in fact converted into both rifamycin $\mathrm{B}^{4)}$ and rifamycin $\mathrm{G}$ by washed mycelium of $N$. medi-

Fig. 1

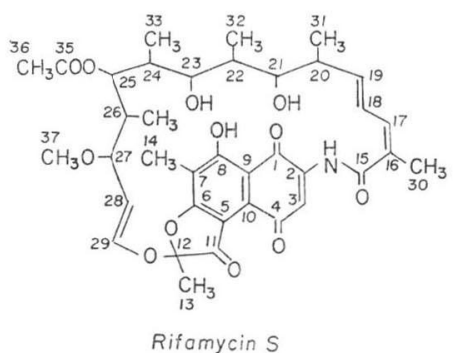

Isolation and Analytical properties

Rifamycin $\mathrm{G}$ is produced together with rifamycin complex in normal fermentations (for conditions and media see for instance ${ }^{4)}$ ) but it first escaped notice since it is biologically inactive.

It was later noticed in extracts because of its ultraviolet absorption at $383 \mathrm{~nm}$ and was obtained as a crude material by:

a) Extraction of the filtered acidified broth with $\mathrm{CHCl}_{3}$.

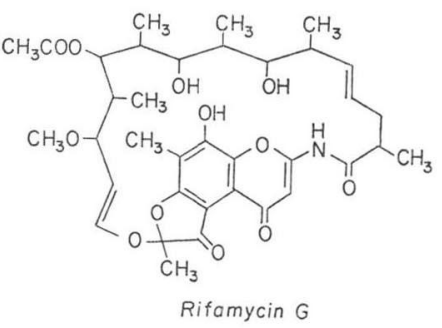

terranei, as shown by the data reported in Table 1. The presence of barbital in the medium appears to increase the conversion into rifamycin $\mathrm{B}$, but to have no effect on rifamycin $G$ yields. Evidence that rifamycin $\mathrm{G}$ does not derive from de novo synthesis was obtained by adding to washed mycelium suspensions $C^{14}$ labelled rifamycin $S(100 \mathrm{mg}$, $5.8 \times 10^{5} \mathrm{dpm} / \mathrm{m}$ mole). After incubation rifamycin $G$ was recovered $(27 \mathrm{mg})$ having a specific activity of $3.6 \times 10^{5} \mathrm{dpm} / \mathrm{m}$ mole. 
Table 1. Transformation of rifamycin $S$ into rifamycins $\mathrm{B}$ and $\mathrm{G}$ by washed $N$. mediterranei mycelium

\begin{tabular}{c|c|c|c|c}
\hline $\begin{array}{c}\text { Barbital } \\
\text { in } \\
\text { growth } \\
\text { medium }\end{array}$ & $\begin{array}{c}\text { Barbital } \\
\text { in resus- } \\
\text { pension } \\
\text { medium }\end{array}$ & $\begin{array}{c}\text { Rifamycin } \\
\text { S added } \\
(\mu \mathrm{g} / \mathrm{ml})\end{array}$ & $\begin{array}{c}\text { Rifamycin } \\
\text { B formed } \\
(\mu \mathrm{g} / \mathrm{ml})\end{array}$ & $\begin{array}{c}\text { Rifamycin } \\
\text { G formed } \\
(\mu \mathrm{g} / \mathrm{ml})\end{array}$ \\
\hline- & - & - & 8 & 17 \\
- & - & 200 & 70 & 77 \\
- & + & - & 13 & 10 \\
- & + & 200 & 89 & 62 \\
+ & + & - & 16 & 10 \\
+ & + & 200 & 114 & 76 \\
\hline
\end{tabular}

Washed mycelium of Nocardia mediterranei was prepared and resuspended in $0.07 \mathrm{M}$ phosphate buffer $\mathrm{pH} 6.5$ as previously described. ${ }^{4)}$ The media differed for the presence $(+)$ or absence $(-)$ of $1 \mathrm{~g} /$ liter of sodium diethylbarbiturate. Where indicated $200 \mu \mathrm{g} / \mathrm{ml}$ of rifamycin $\mathrm{S}$ were added to the resuspended mycelium. After 20 hours of incubation at $28^{\circ} \mathrm{C}$ the products were analyzed. Rifamycin B was determined by a spectrophotometric differential method ${ }^{7}$ and rifamycin $G$ was estimated spectrophotometrically after extraction and counter-current distribution.

\section{Structural Considerations}

Elemental analysis and high resolution mass spectrometry show that rifamycin $G$ has two hydrogen atoms more and one carbon atom less than its parent rifamycin $\mathrm{S}$.

Comparison of ${ }^{1} \mathrm{H}$ NMR spectra of the two compounds (Fig. 2; for rifamycin $\mathrm{S}{ }^{1} \mathrm{H}$ NMR spectrum $\mathrm{see}^{8)}$ ) established that the two extra hydrogen atoms are located on carbon 16 and 17. In fact the saturation of the $16-17$ double bond of rifamycin $\mathrm{S}$ is demonstrated by the disappearance of the singlet $(3 \mathrm{H})$ at $\sigma=2.01$ (attributed to methyl 30 at C-16) and the appearance of a new doublet $(3 \mathrm{H})$ at $\delta=$ 1.30. Moreover the signal of the hydrogen at $\mathrm{C}-17$ is no more present in the region $\delta=$ $6.0 \sim 6.5$ and the signals corresponding to hydrogens on carbon 18 and 19 (that are now on a isolated double bond) are shifted upfield at $\delta=5.4 \sim 5.8$.

Substantially unchanged are the chemical shifts of the signals corresponding to methyl 37 (methoxy at $\mathrm{C}-27, \delta=3.10$ ) methyl 36 (acetoxy at C-25, $\delta=2.02$ ), hydrogens on double bond $28-29(\delta=5.25$ and $\delta=6.29)$, hydrogens on oxygen bearing carbons $25(\delta=4.87)$, $23(\delta=3.04), 21$ and $27(\delta=3.2 \sim 3.6)$. This suggests that no other variation occurred in the ansa chain structure; moreover the chemical shifts of the methyls $31,32,33,34$ (doublets from $\delta=0.1$ to 1 ) demonstrate that, as in rifamycin $\mathrm{S}$ the ansa chain is shielded by

Fig. 2. ${ }^{1} \mathrm{H}$ NMR spectrum of rifamycin $\mathrm{G}$.

The spectrum was recorded at $60 \mathrm{MHz}$ in $\mathrm{CDCl}_{3}$, concentration $3 \times 10^{-2} \mathrm{M}$, PFT NMR, Bruker WT60.

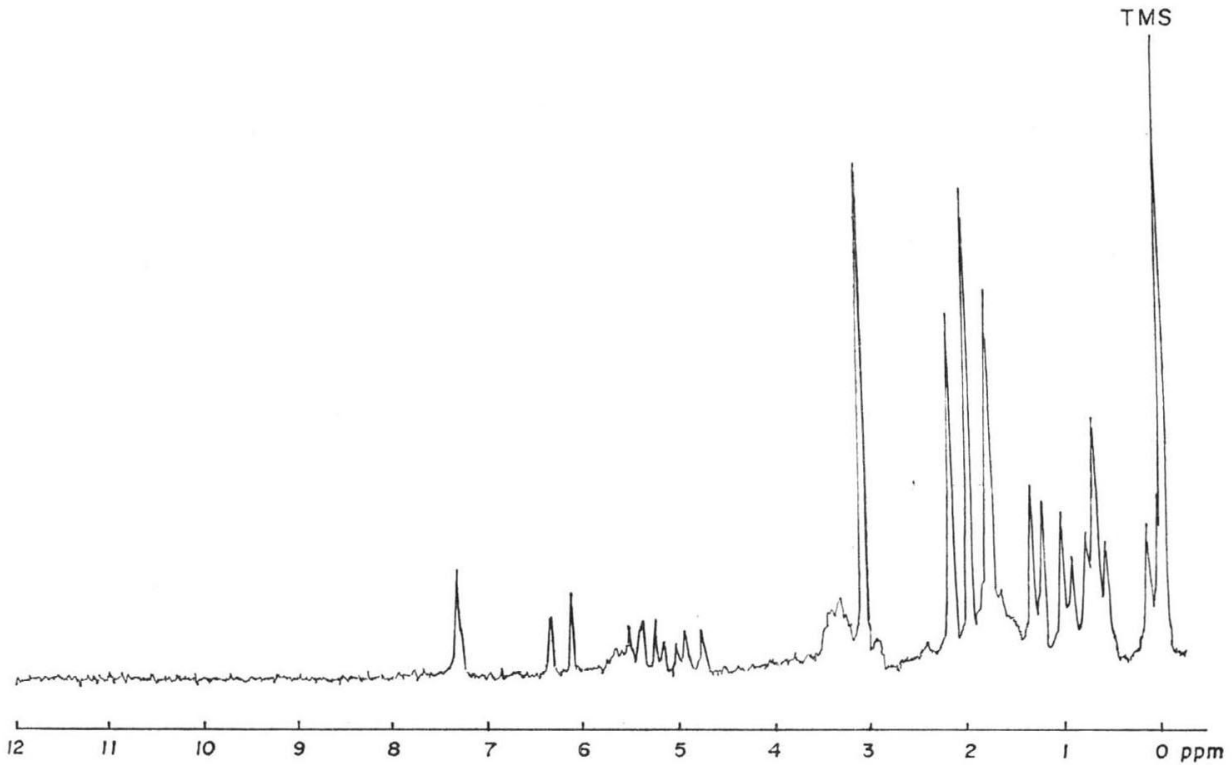


the aromatic ring and thus still spans it. This is supported also by the IR spectrum where the peaks corresponding to $\nu \mathrm{C}=\mathrm{O}$ of the acetyl group at $1720 \mathrm{~cm}^{-1}$ and to the second amide band $\delta \mathrm{N}-\mathrm{H}$ at $1510 \mathrm{~cm}^{-1}$ could be identified. ${ }^{\text {) }}$

The u.v. spectrum indicates a major modification in the naphthoquinone moiety, the socalled chromophore. Comparison of the electron impact mass spectrum of rifamycins $G$ and $\mathrm{S}^{102}$ revealed that the high intensity peak at $m / e 273\left(\mathrm{C}_{14} \mathrm{H}_{11} \mathrm{NO}_{5}\right)$ of rifamycin S attributed to the chromophoric ion was replaced by a peak at $m / e 261 \quad\left(\mathrm{C}_{13} \mathrm{H}_{11} \mathrm{NO}_{5}\right)$. Conversion of rifamycin $\mathrm{S}$ to rifamycin $\mathrm{G}$ thus involves the loss of one carbon atom in the chromophore. No variation in respect to rifamycin S appears to have occurred in the aromatic and furanone rings including carbon atoms from 5 to 12 . In fact ${ }^{1} \mathrm{H}$ NMR signals of methyl $14(\delta=2.20)$ and methyl $13(\delta=1.80)$ are substantially unchanged. In the I.R. spec trum the $\nu \mathrm{C}=\mathrm{O}$ of furanone carbonyl is still present at $1725 \mathrm{~cm}^{-1}$. The hydroxyl group on carbon 8 appears (as usual in rifamycins) to be responsible for the acidity of the molecule: on treatment with diazomethane a monomethyl derivative is obtained (m.p. 248 $250^{\circ} \mathrm{C}$, analysis and molecular ion in agreement for $\mathrm{C}_{37} \mathrm{H}_{49} \mathrm{NO}_{12}$ ) in which only the weak acidic function (pK 10.5) is retained and whose ${ }^{1} \mathrm{H}$ NMR shows a signal (singlet, $3 \mathrm{H}$ ) at $\delta=4.10$ superinposable with that attributed to the aromatic methoxy of 8-methyl rifamycin $\mathrm{S}$.

One of the carbon atoms 1 to 4 of rifamycin $\mathrm{S}$ is thus missing in rifamycin G. Several evidences indicate that it might be the carbon atom 1; in fact the carbonyl group in this position is no more present in rifamycin $G$ since: a) the molecule lacks oxido-reduction properties; b) in rifamycin $\mathrm{S}$ the hydroxyl group in position 8 gives a strong hydrogen bond with the carbonyl group in position 1 as shown by a signal at $\delta 12.51$ in ${ }^{1} \mathrm{H}$ NMR. No such signal is present in rifamycin $G$ spectrum; c) the lack of this hydrogen bond explains the lower pKa value (3.1) of rifamycin $\mathrm{G}$ in comparison with rifamycin $\mathrm{S}(\mathrm{pKa}=7.2)$; d) in ${ }^{1} \mathrm{H}$ NMR spectrum the signal attributed to the hydrogen on carbon 3 is shifted from $\delta$ 7.77 of rifamycin $\mathrm{S}$ to $\delta=7.35$ in rifamycin $\mathrm{G}$.
This suggests that in the latter it is no more in $\beta$ position to a carbonyl group.

In conclusion, the structure reported in Fig. 1 in which the quinone ring of rifamycin $\mathrm{S}$ is replaced by a $\gamma$-pyrone ring is consistent with all the data above discussed and is thus proposed for rifamycin $\mathrm{G}$.

\section{Acknowledgement}

The authors wish to thank Dr. G. G. Gallo and his associates for physico-chemical,determinations and in particular Dr. E. Martinelli for help in NMR interpretation.

\section{References}

1) Sensi, P.; A. Greco \& R. Ballotta: Isolation and properties of rifamycin $B$ and rifamycin complex. Antibiotics Annual 1959: 262 270, 1960

2) Lancini, G. C. \& C. Hengheller: Isolation of rifamycin SV from a mutant Streptomyces mediterranei strain. J. Antibiotics 22: 637 638, 1969

3) White, R. J.; E. Martinellt \& G. C. LANCINI: Ansamycin biogenesis: Studies on a novel rifamycin isolated from a mutant strain of Nocardia mediterranei. Proc. Nat. Ac. Sci. 71: 3260 3264, 1974

4) Lancini, G. C.; G. G. Gallo, G. Sartori \& P. SENSI: Isolation and structure of rifamycin L and its biogenetic relationship with other rifamycins. J. Antibiotics 22: 369 377, 1969

5) Sugawara, S.; K. Karasawa, M. Watanabe \& T. Hidaka: Production of rifamycin $O$ by Streptomyces $4107 \mathrm{~A}_{2}$. J. Antibiotics, Ser. A 17: 29 32, 1964

6) Birner, J.; P. R. Hodgson, W. R. Lane \& E. H. BAXTER: An australian isolate of Nocardia mediterranei producing rifamycin SV. J. Antibiotics 25: 356 359, 1972

7) Pasqualucci, C. R.; A. Vigevani, P. Radaelli \& G. G. Gallo: Improved differential spectrophotometric determination of rifamycins. J. Pharm. Sci. 59: 685 687, 1970

8) Gallo, G. G.; E. Martinelli, V. Pagani \& P. SENSI: The conformation of rifamycin $\mathrm{S}$ in solution by ${ }^{1} \mathrm{H}$ NMR spectroscopy. Tetrahedron 30: 3093 3097, 1974

9) Ferrari, P. \& G. G. Gallo: Infrared spectra of rifamycins. I1 Farmaco, Ed. Sc. 30: 676 696, 1975

10) Zerillil, L. F.; M. LANDi, N. Rimorini \& G. G. Gallo: Spettri di massa delle rifamicine. Annali di Chimica 64: 199 206, 1974 